\title{
STEM stacking fault analysis in Ni-based super alloys
}

\author{
P.J. Phillips ${ }^{1}$, M. De Graef ${ }^{2}$, and M. Mills ${ }^{1}$
}

${ }^{1}$ Department of Materials Science and Engineering, Ohio State University, Columbus OH 43210

2 Department of Materials Science and Engineering, Carnegie Mellon University, Pittsburgh PA 15213

Transmission electron microscopy (TEM) has been one of the most influential tools in advancing the field of crystal defect analysis. Conventional TEM (CTEM) imaging modes, such as bright field (BF), dark field (DF), and weak beam dark field (WBDF or $\mathbf{g - 3}$ ) have been well-documented in the scientific literature, and simulation methods are readily available for each. The advent of the field emission gun (FEG) has benefitted chemical analysis and atomic-scale imaging of materials via the high angle annular dark field (HAADF) observation mode (see, for example [1]). The present contribution highlights the use of a FEG-TEM, operated in scanning transmission electron microscopy (STEM) mode, as a viable tool for defect analysis.

STEM holds many advantages over CTEM, for instance thicker specimens are more receptive to STEM imaging, while it also tends to suppress bend contours and other contrast effects [2,3]. In the 1970s, STEM looked to be emerging as a promising technique, with successful instances of STEM imaging of crystalline defects $[2,4,5]$. It is noted that in STEM mode, traditional $\mathbf{g} \cdot \mathbf{b}$ and $\mathbf{g} \cdot \mathbf{R}$ invisibility criteria are valid, and the principal of reciprocity is often considered when analyzing the resulting contrast [6]. However, one must be cautious in applying these principles, as they are only valid under stringent microscope settings, often not representative of ideal imaging conditions [3]. Hence, there is a need for proper STEM defect image simulations to analyze experimental results.

A stacking fault (SF) present in a precipitation-strengthened Ni-based superalloy was examined using both CTEM and STEM diffraction contrast methods. CTEM imaging was performed on a Philips CM-200T operated at $200 \mathrm{kV}\left(\mathrm{LaB}_{6}\right.$ cathode) and all STEM imaging was performed on an FEI Tecnai F20 field emission $200 \mathrm{kV}$ S/TEM. Image simulations were carried out using a dynamical systematic row algorithm; the algorithm is described in a separate contribution at this conference [7].

Fig. 1(a) shows conventional bright field/dark field (BF/DF) experimental images (left column) of an extrinsic stacking fault with displacement vector $\mathbf{R}=\frac{1}{3}[1 \overline{1} \overline{1}]$ on the $(\overline{1} 11)$ plane. Seven-beam systematic row image simulations, using the scattering matrix approach, are shown in the right column; the experimental images show some variations in the fringe spacing, due to minor foil thickness variations, and the vertical thin white line indicates where the agreement between experiment and simulation is satisfactory. STEM images, using a diffraction aperture to select a small region on the BF detector, are shown in Fig. 1(b) for $\mathbf{g}=(11 \overline{1})$ and $\mathbf{g}=(\overline{1} \overline{1})$ operative reflections. The simulated images correspond to a weighted average of 51 individual images for a range of incident beam directions, and are in good agreement with the observations. When the diffraction aperture is removed, the images of Fig. 1(c) are obtained; the stacking fault fringes are clearly visible near the top of the foil but become blurred towards the bottom, because both the transmitted beam and several diffracted beams contribute to the BF detector signal. The simulated images are, once again, in good agreement with the observations. It should be noted that no attempt was made to match the bounding partials of this stacking fault. The effects of diffraction aperture position as well as other microscope parameters, such as camera length, convergence angle, and detector acceptance angle, will be discussed in detail. The ability to obtain interpretable diffraction contrast images via STEM that are of comparable quality to CTEM images, and the new simulation capabilities, contribute to establishing a basis for STEM defect imaging and interpretation as a viable method of crystalline defect analysis. 


\section{References}

[1] J.M. Cowley, in Handbook of Microscopy for Nanotechnology, eds. N. Yao and Z.L. Wang, (2005).

[2] K.E. Easterling, J. Mater. Sci, 12 (1977) 857-868.

[3] G.R. Booker, et al., Proc. of the $7^{\text {th }}$ SEM Symposium, eds. O. Johari and I. Corvin (1974) 225-234.

[4] G.R. Booker, et al., Proc. of the $6^{\text {th }}$ SEM Symposium, eds. O. Johari and I. Corvin (1973) 251-258.

[5] D.M. Maher and D.C. Joy, Ultramicroscopy, 1 (1976) 239-253.

[6] C.J. Humphreys, Ultramicroscopy, 7 (1981) 7-12.

[7] M. De Graef, Proceedings of this conference (2010).

[8] Part of this work was supported by the National Science Foundation under grant \# DMR-0809048.

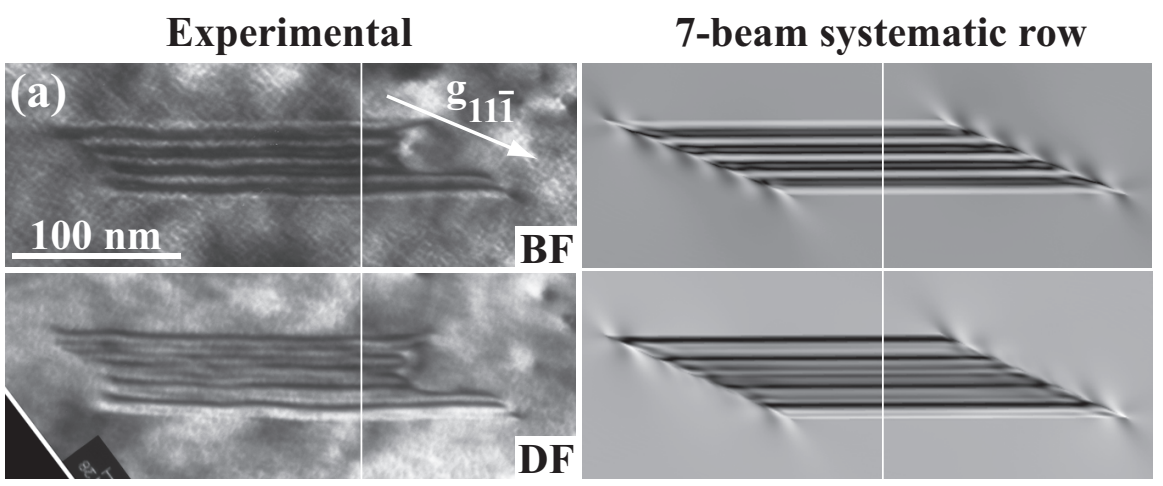

CTEM multi-beam simulation

Beam direction [112]

Foil normal [112], thickness $128 \mathrm{~nm}$

Stacking fault $R=\left[\begin{array}{lll}1 & -1 & -1\end{array}\right] / 3$

Stacking fault plane (-1 11 )

Bragg orientation for $g=\left(\begin{array}{lll}1 & 1 & -1\end{array}\right)$
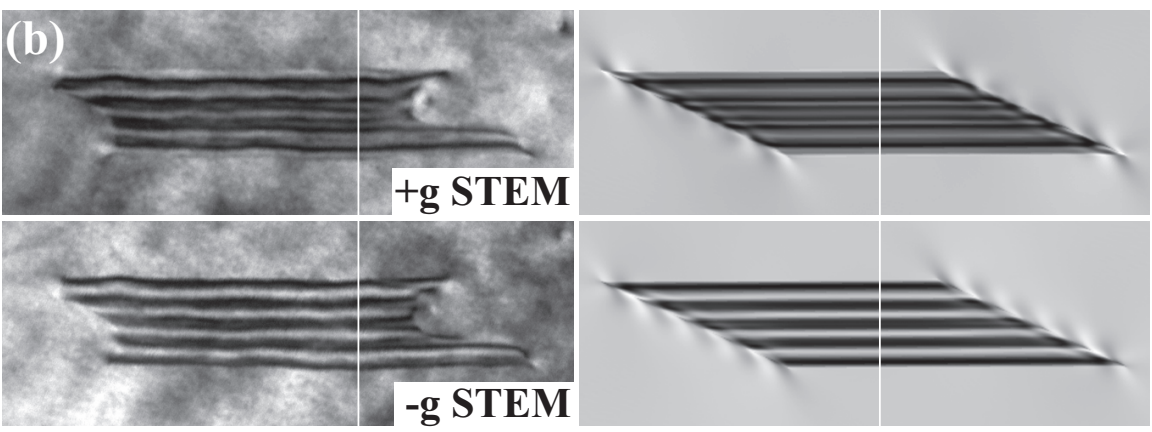

STEM multi-beam simulation

Same conditions as above +

$\mathrm{BF}$ detector radius $3.5 \mathrm{~mm}$

Camera length $114 \mathrm{~mm}$

Beam convergence angle $8 \mathrm{mrad}$

Diffraction aperture radius $2.65 \mathrm{mrad}$

51 sampling beam orientations
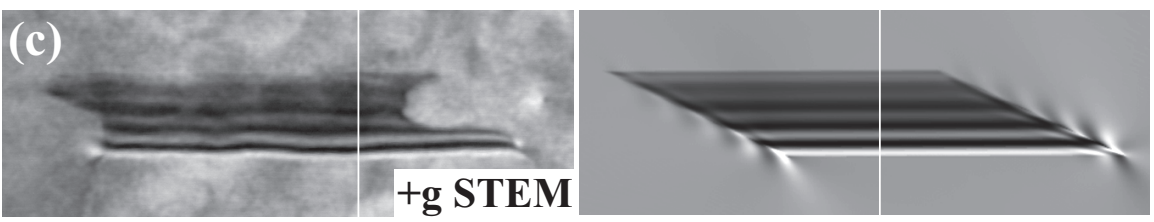

STEM multi-beam simulation

Same conditions as in (b) except that the diffraction aperture was removed from the beam path.
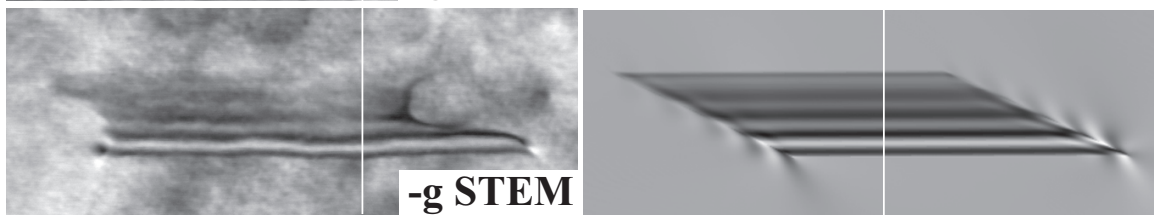

Figure 1: Experimental (left column) and simulated (central column) 7-beam systematic row stacking fault images for the conditions described in the text and in the rightmost column. 